\title{
Numerical Simulation and Analysis of the Coupled Flow Field of Multiple Propeller-Type Current-Meters Based on CFD
}

\author{
Yi-yong Yao ${ }^{1}$, Zhong-hai $\mathrm{Yu}^{1+}$ and Li-ping Zhao ${ }^{2}$ \\ ${ }^{1}$ School of Mechanical Engineering, Xi'an Jiaotong University, Xi'an, P.R.China \\ ${ }^{2}$ State Key Laboratory for Manufacturing Systems Engineering, Xi'an Jiaotong University, Xi'an, P.R.China
}

\begin{abstract}
The standard k- $\varepsilon$ turbulence model in computational fluid dynamics software FLUENT was used, and the coupled flow field of multiple propeller-type current-meters was simulated numerically, properties of the coupled flow field under different distances between each two current-meters and different initial flow velocity were found out. Results show that: the interaction on flow field of the adjacent current-meters causes the bimodal trend of flow velocity which means flow velocity decreases rapidly after the peak; the rod deformed in the flow field along flow direction and make the deflection of axes of current-meters happen, which cause the deviation between measured velocity and actual velocity, and this deviation became larger with the flow velocity increases. The detailed information of flow field is obtained in this paper, which provides theoretical evidence to development and actual measurement of propeller-type current-meter.
\end{abstract}

Keywords: CFD, coupled flow field, propeller-type current-meter, numerical simulation.

\section{Introduction}

The measurement of the velocity of water flow is very important to the study of the movement law of water flow and the interaction mechanism of water and sediment [1-4]. At home and abroad, the research on propeller-type current-meter has made some progress in theory and engineering application. Computational Fluid Dynamics (CFD) as an independent discipline, has become a hot research topic in fluid mechanics [5]. In summary, based on the gap of Study on the coupling flow field of multiple current-meters, use FLUENT module in CFD software ANSYS to simulate the flow field of multiple propeller-type current-meters, and combined with the static analysis module of ANSYS-Static Structural, the deformation of current-meters in the flow field is obtained.

\section{Flow Flied Modelling}

\subsection{Hydrodynamic equation}

Under the condition of natural hydrology, it is believed that the flow around the current-meter is a viscous incompressible fluid motion. Based on the Reynolds equation theory, continuity equation and momentum equation of flow field are:

$$
\begin{gathered}
\frac{\partial u_{i}}{\partial x_{i}}=0 \\
u_{j} \frac{\partial u_{i}}{\partial x_{j}}=-\frac{1}{\rho} \frac{\partial p}{\partial x_{i}}+v \frac{\partial^{2} u_{i}}{\partial x_{j} \partial x_{j}}-\frac{\partial \overline{u_{i}^{\prime} u_{j}^{\prime}}}{\partial x_{j}}+f_{i}
\end{gathered}
$$

where: $\rho$-Liquid density

$u_{i}^{\prime}, u_{j}^{\prime}$-Pulsation quantity

$x_{i}, x_{j}$-Coordinate variable of Descartes coordinate system 
$p$-Reynolds static pressure

$f_{i}$-The component of the mass flow strength on the coordinate axis

In order to solve the flow field in rotating region, turbulence model is needed. In the engineering calculation of turbulent flow, the standard $\mathrm{k}$ - e double equation model is the most widely applied, its $\mathrm{k}$ equation and $\varepsilon$ equation are:

$$
\begin{gathered}
\frac{\partial}{\partial x_{j}}\left(\rho u_{j} k\right)=\frac{\partial}{\partial x_{j}}\left(\frac{\mu_{e f f}}{\sigma_{k}} \frac{\partial k}{\partial x_{j}}\right)+\mathrm{G}-\rho \varepsilon \\
\frac{\partial}{\partial x_{j}}\left(\rho u_{j} \varepsilon\right)=\frac{\partial}{\partial x_{j}}\left(\frac{\mu_{e f f}}{\sigma_{\varepsilon}} \frac{\partial \varepsilon}{\partial x_{j}}\right)+\frac{\varepsilon}{k}\left(C_{1} G-C_{2} \rho \varepsilon\right)
\end{gathered}
$$

where: $k$-Turbulent kinetic energy

$\mathrm{G}$ - Turbulent kinetic energy

$\mu_{\text {eff }}$-Effective viscosity coefficient $\varepsilon$ - dissipation rate

The constants in the equation are: $\sigma_{k}=1.0, \sigma_{\varepsilon}=1.3, C_{1}=1.44, C_{2}=1.92$.

\subsection{Geometry modelling and mesh partition}

Geometric model of a single propeller-type current-meter shows in Fig. 1. After pre-processing the model in ANSYS, import the three flow meter models in different distances. The whole flow field is meshed in Tetrahedrons method. In order to improve the precision of calculation, the refinement of the mesh in the region is necessary. Surface mesh of current meter shows in Fig. 2.

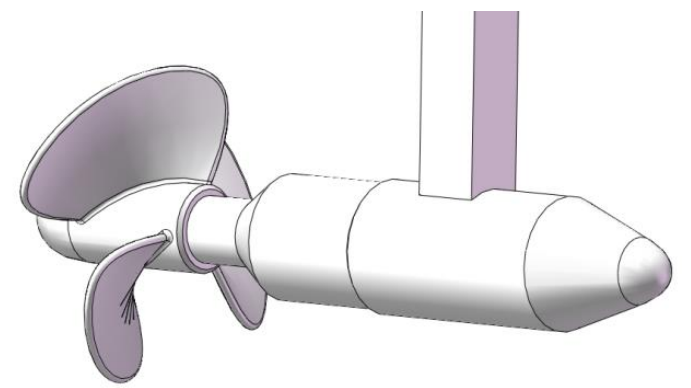

Fig. 1: Geometric model of single current-meter

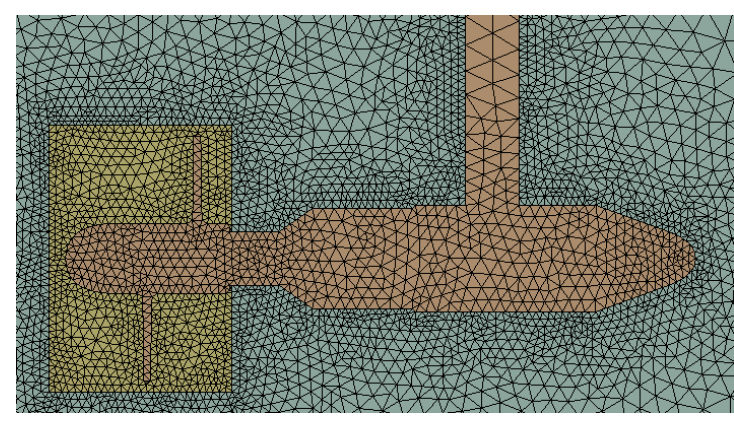

Fig. 2: Flow field mesh model

\subsection{Boundary conditions and parameter settings}

In this paper, the fluid medium is liquid water. Flow inlet boundary is velocity inlet, Under the same spacing of current-meters, the different inlet water velocity is set. The initial velocity of water flow and the rotational speed of rotating flow field show in Table.1; Using SIMPLE algorithm for calculating, and turbulent momentum equation, turbulent kinetic energy equation, turbulent dissipation rate equation are in second order upwind format.

\begin{tabular}{|c|c|c|c|c|}
\hline \multirow{2}{*}{$\begin{array}{c}\text { Flow } \\
\text { parameters }\end{array}$} & \multicolumn{4}{|c|}{ Simulation scheme } \\
\hline & 1 & 2 & 3 & 4 \\
\hline $\begin{array}{c}\text { Flow velocity } \\
(\mathrm{m} / \mathrm{s})\end{array}$ & 2 & 4 & 6 & 8 \\
\hline $\begin{array}{c}\text { Rotational speed } \\
\text { (rpm) }\end{array}$ & 500 & 1000 & 1500 & 2000 \\
\hline
\end{tabular}

Table 1: Flow parameters setting

\section{Calculation Results and Analysis}

\subsection{Analysis of flow field of single propeller-type current-meter}

The flow field of a single flow meter is analysed, with the flow velocity of $6 \mathrm{~m} / \mathrm{s}$, the velocity contour of the flow field shows in Fig. 3. Set the reference line at the distance of $50 \mathrm{~mm}, 62.5 \mathrm{~mm}, 75 \mathrm{~mm}, 87.5 \mathrm{~mm}$, $100 \mathrm{~mm}$ from current-meter, the flow velocity curve on the reference line show in Fig.4. 


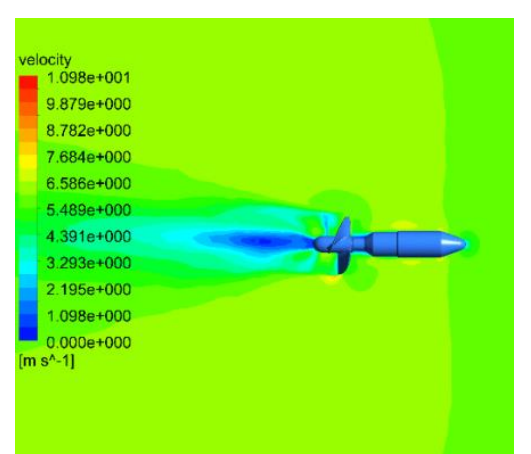

Fig. 3: Velocity contour of the flow field

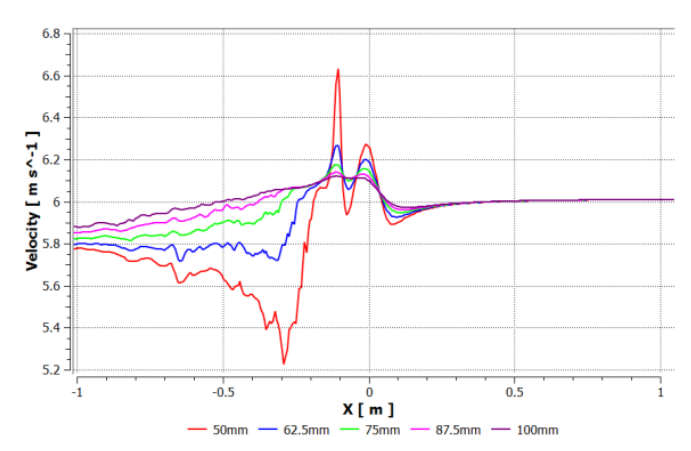

Fig. 4: Flow velocity curve on the reference line

It can be seen from Fig. 3 and Fig. 4: the velocity of the flow around the flow meter is higher than other area but not too much; the smaller the distance is, the stronger the flow field is affected by the current meter, which means that before the first peak of curve the flow velocity increase more and in the second peak of curve the flow velocity decreases rapidly.

\subsection{Analysis of the coupled flow field of multiple propeller-type current-meters}

When the initial flow velocity is $6 \mathbf{m} / \mathbf{s}$, and the spacing of current-meters are $100 \mathrm{~mm}, 125 \mathrm{~mm}, 150 \mathrm{~mm}$, $175 \mathrm{~mm}, 200 \mathrm{~mm}$, velocity contour of flow field on plane of current-meters show in the Fig. 5. Through comprehensive analysis of this five velocity contours, we can find that the presence of multiple flow meters has a great influence on the flow field.

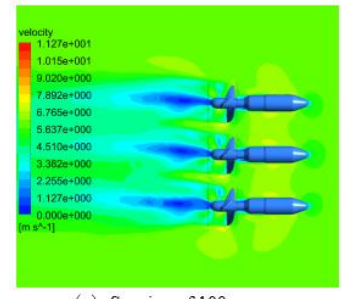

(a) Spacing of $100 \mathrm{~mm}$

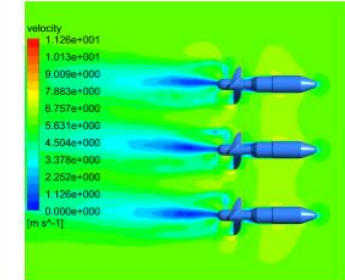

(b) Spacing of $125 \mathrm{~mm}$

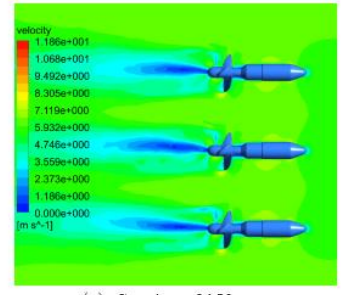

(c) Spacing of $150 \mathrm{~mm}$

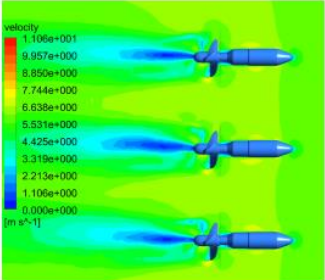

(d) Spacing of $175 \mathrm{~mm}$

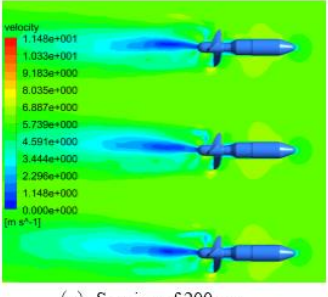

(e) Spacing of $200 \mathrm{~mm}$

Fig. 5: Velocity contour of flow field

In order to reflect the influence of flow field more directly, a reference line is arranged at the middle position of the adjacent current-meter, as shown in Fig. 6, and the change trend of velocity in the straight line is obtained. Compared with the velocity variation of the single current-meter at the same position, we can get the curves of flow velocity under different distances.

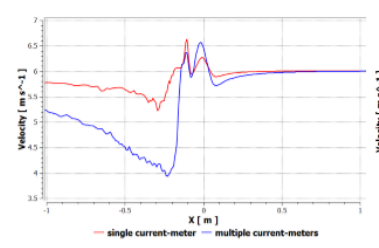

(a) Spacing of $100 \mathrm{~mm}$

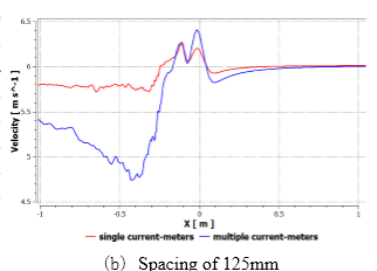

(b) Spacing of $125 \mathrm{~mm}$

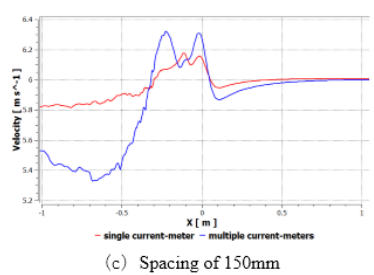

(c) Spacing of $150 \mathrm{~mm}$
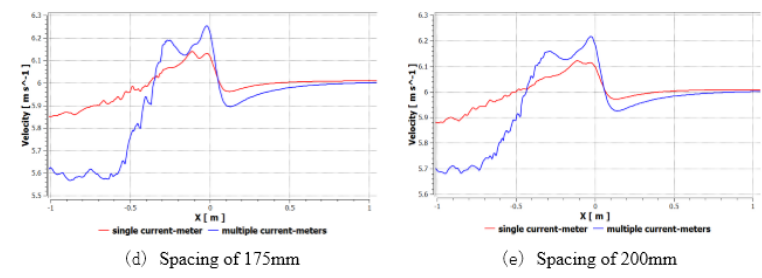

Fig. 6: Curves of flow velocity 
It can be seen from Fig. 6, under the coupling of the flow field of the adjacent current-meter, the trend of the velocity on the reference line has changed greatly compared with the single current-meter condition. In order to show the two peak's characteristics, import a k value to express reduced rate of the flow velocity. Its computation formula is:

where: $\quad V_{\max }$ - peak velocity

$$
\mathrm{k}=\frac{V_{\max }-V_{\min }}{x_{\max }-x_{\min }}
$$

$V_{\text {min }}$-lowest velocity

$x_{\max }$-abscissa of peak velocity

$x_{\text {min }}$-abscissa of lowest velocity

The $\mathrm{k}$ value can reflect the influence of the flow field coupling on the flow velocity, the larger the $\mathrm{k}$ value is, the faster the flow velocity decreases after the peak; on the other hand, the smaller $\mathrm{k}$ value is, the slower the flow velocity decreases after the peak. The relationship between the $\mathrm{k}$ value and the spacing of the current-meter in the decline of the second peak shows in the Fig. 7; the relationship between the k value and the spacing of the current-meter in the decline of the first peak shows in the Fig. 8.

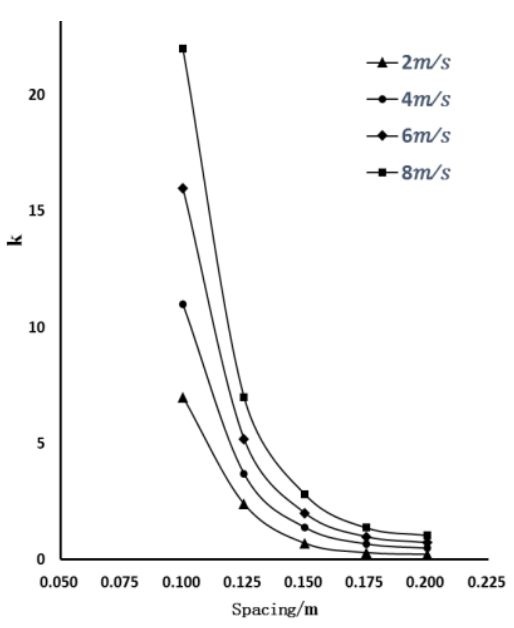

Fig. 7: The relationship of the second peak

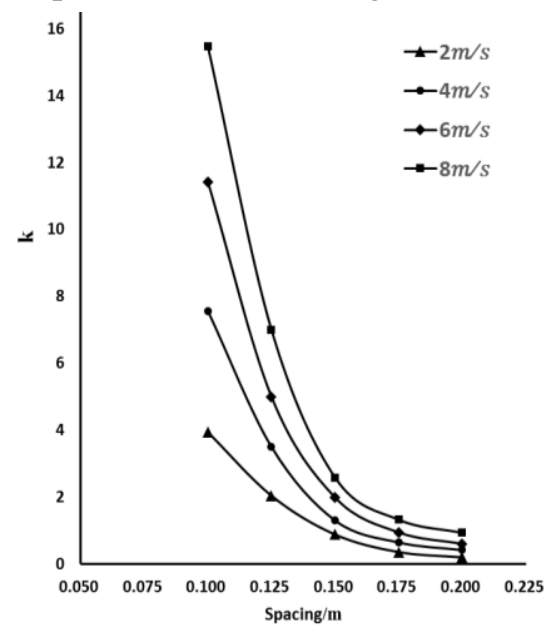

Fig. 8: The relationship of the first peak

After separate analysis of Fig. 7, Fig. 8, we can get: the k value decreases with the increase of the spacing, and it is inversely proportional to the spacing, which means that the coupling effect between the flow field of the adjacent current-meter decreases with the increase of the spacing of the adjacent flow meters, and when the spacing is reduced to a certain value, this influence will have a leaping growth.

\subsection{Analysis of the deformation of current-meter}

When the initial velocity are $2 \mathrm{~m} / \mathrm{s}, 4 \mathrm{~m} / \mathrm{s}, 6 \mathrm{~m} / \mathrm{s}, 8 \mathrm{~m} / \mathrm{s}$, the deformation of current-meter shows in Fig.9. The relationship between the maximum deformation of current-meter shows in Fig. 10.

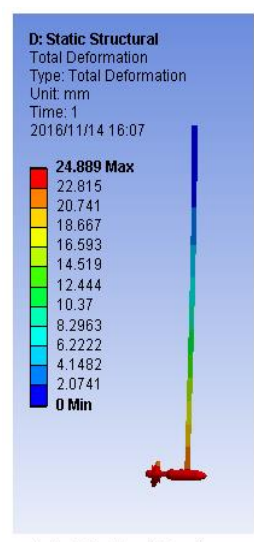

(a) Velocity of $2 \mathrm{~m} / \mathrm{s}$

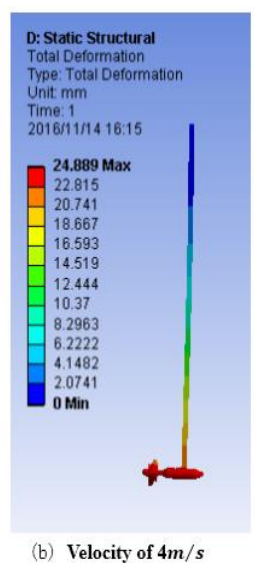

(b) Velocity of $4 \mathrm{~m} / \mathrm{s}$

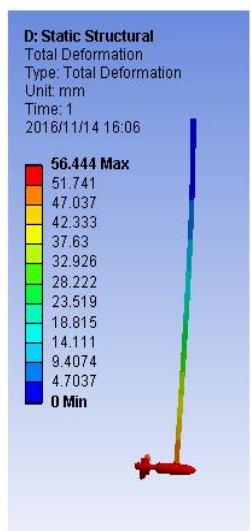

(c) Velocity of $6 \mathrm{~m} / \mathrm{s}$

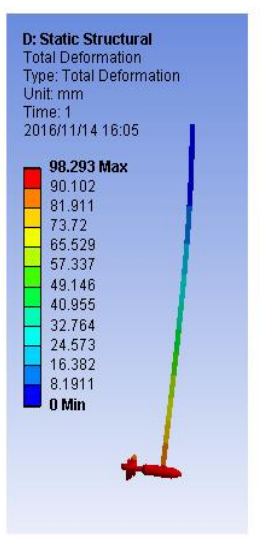

(d) Velocity of $8 m / s$

Fig. 9: the deformation of current-meter

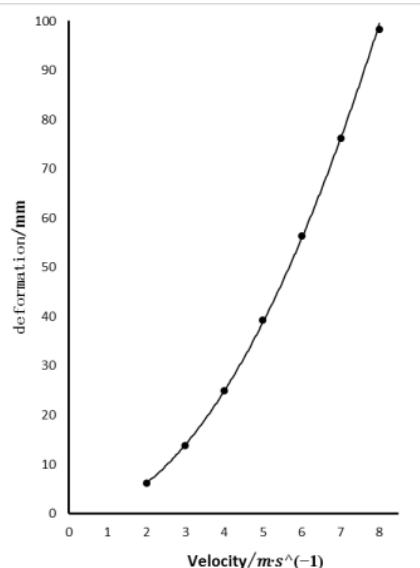

Fig. 10: the relationship 
From Fig. 10 we can get the formula of the maximum deformation of the current meter:

$$
X=K \cdot V^{2}
$$

where $\mathrm{X}$ - the maximum deformation, $\mathrm{V}$ - the initial velocity of flow field

$\mathrm{K}$ is a constant value, the specific value of $\mathrm{K}$ depends on the shape and size of the current meter, in this paper $\mathrm{K}=1.55$. The deformation of rod causes the deformation of the current-meter, and the impact of water flow can be regarded as uniform, so the rod is simplified as the cantilever beam model with uniform pressure. The formula for calculating the deflection angle of current-meter is:

$$
\theta=\frac{8}{6 L} \cdot X=\frac{8 K \cdot V^{2}}{6 L}
$$

where $\theta$ - deflection angle of current-meter, $\mathrm{L}$ - length of rod

The current-meter axis is not parallel to the flow direction because of the deflection. Therefore, the actual flow velocity measured by the current meter is:

$$
V^{\prime}=V \cdot \cos \theta=V \cdot \cos \frac{8 K \cdot V^{2}}{6 L}
$$

$V^{\prime}$ is the actual flow velocity measured by the current meter. The formula we can get: with the increase of the initial velocity, the difference between the measured velocity and the accurate velocity is larger, which means the error is larger.

\section{Conclusion}

Through the numerical simulation and analysis of the coupled flow field of multiple propeller-type current-meters, the detailed information of flow field is obtained, which provides theoretical evidence to development and actual measurement of propeller-type current-meter. Next, the experiments of actual behaviours of multiple propeller-type current-meters underwater are needed, in order to verify the accuracy of the simulation results obtained in this paper.

\section{Acknowledgment}

This work is supported by National Scientific \&Technological Major Projects (2012ZX04002-071).

\section{References}

[1] Wen Hua, Zhu Yu-chen, Fu Guang. The Research of Flowing Velocity Instrument at Home and Abroad. In: Chemical Defense on Ships. 2007, (03):43-45.

[2] Jin Fu-yi, Design of Signal Acquisition System of Current Meter. Journal of China Hydrology. 2016,(01):88-91+36.

[3] Wang Hao, C hen Huai, Li Dan-xun, Wang Xing-ku. Impact of horizontal plug-in flow meters on the velocity field-An experimental study. J Tsinghua Univ. (ScI \& Technol). 2016,(12):1271-1277.

[4] Lv Shou-gui, Discussion on unconventional current meter method for flow measurement in high flood period. Heilongjiang Science. 2016,(08):36-37.

[5] Wu Li-chun. Application Progress of FLUENT Software in the Hydraulics. Journal of Chongqing College of Education. 2009,(03):66-68. 